\title{
Diagnostic Value of the Mean Platelet Volume in the Prediction of Respiratory Syncytial Virus in Acute Bronchiolitis
}

\author{
Şule Gökçe1, Zafer Kurugöl2, Güldane Koturoğlu2, Aslı Aslan3, Sibel Polater1, Candan Çiçek4
}

${ }^{1}$ Ege University Faculty of Medicine, Department of Pediatrics, General Pediatrics Unit, İzmir, Turkey

2Ege University Faculty of Medicine, Department of Microbiology, İzmir, Turkey

\begin{abstract}
Aim: Respiratory Syncytial virus (RSV) is an important pathogen of childhood that causes lower respiratory system infection. The aim of this study was to evaluate whether the mean platelet volume (MPV) changes are significant in the predietion of bronchiolitis with respiratory syncytial virus (RSV) and other respiratory viruses.

Materials and Methods: A standard protocol was followed, and 184 patients were divided into groups based on being RSV positive and other respiratory viruses positive. Using the ROC, diagnostic accuracy was evaluated according to the AUC for the diagnosis of bronchiolitis. A P value of $<0.05$ was considered statistically significant.

Results: MPV was significantly lower in patients with single RSV $(6.6 \pm 1.1 \mathrm{Vs} .7 \pm 1.2, P<0.05)$. The MPVs were similar in patients with positive and negative RSV bronchiolitis $(6.8 \pm 1.5$ vs. $7 \pm 1.3, p>0.05)$ and other viruses. ROC curve analysis suggested that MPV level cut-off point for making the diagnosis of single RSV bronchiolitis was $6.63 \mathrm{fL}$ with a sensitivity, specificity of $55 \%, 63 \%$ respectively. The median AUC was 0.384 for the MPV ( $95 \%$ Cl 0.270-0.499, P=0.04)

Conclusion: Mean platelet volume may be a useful marker to provide a prediction on single Respiratory syncytial virus bronchiolitis. However, measurement of mean platelet volume might not be correct and sufficient to provide a prediction on type of respiratory viruses in bronchiolitis. Keywords: Mean platelet volume, acute bronchiolitis, respiratory syncytial virus, prediction
\end{abstract}

\section{Introduction}

Respiratory Syncytial virus (RSV) is an important pathogen of chitdhood that causes lower respiratory system infection. It is a major burden for children and is associated with over 30 million new acute lower respiratory infection episodes in children under 5 years of age. It gives rise to more than 3.4 million hospital admissions and 160000 deaths every year $(1,2)$. Various studies have shown that RSV is the most common etiologic pathogen with a rate of $50 \%$ to $80 \%$
(3). Almost $30 \%$ to $40 \%$ of cases related to other viruses such as adenovirus, coronavirus, parainfluenza, influenza, rhinovirus, human metapneumovirus, human bocavirus, and human coronaviruses have also been reported as possible pathogens causing acute bronchiolitis (4). Today, various viral diagnostic tests allow us to examine the epidemiological differences/clinical characteristics of respiratory viruses and to provide information of their presence. Many different medical researches based on different diagnostic and predictive strategies are currently being developed to foresee 
RSV-related acute bronchiolitis that is likely to have high mortality and morbidity.

Thrombocytes produced from megakaryocytes in the bone marrow play an important role not only in hemostasis, but also in inflammation, allergic reactions, angiogenesis, repair and renewal of tissues. They also contain mediators such as chemokines, cytokines and coagulation factors which cause strong inflammatory response and tissue regeneration. During any inflammation, production of the thrombocyte increases in the bone marrow and thrombocytes change their volume and distribution range by inflammatory response (5). This thromboctye volume is called mean platelet volume (MPV) in laboratory records. In recent years, several studies have been stated to evaluate the MPV as a biochemical marker in chronic and / or acute inflammatory diseases, and infections (6-8). Increased MPV was described to determine infectious diseases as a marker, whereas several newer studies have revealed that decreased MPV also appeared to be a useful measurement for acute bronchiolitis (9-12). In a study conducted by Renshaw AA et al., it was noticed that some patients infected with RSV had relatively low MPVs (12). However, there are only a few studies that have examined the effects of MPV in acute bronchiolitis, and due to the contraversial results, the clinical significance of MPV in RSV or other viruses-related bronchiolitis has not been clearly defined yet.

The purpose of this study was to evaluate the changes in MPV on hospitalized patients diagnosed with RSV bronchiolitis, to determine whether the MPV could be a predictive marker in RSV bronchiolitis, and also to identify whether the type of viral infection affects MPVs in bronchiolitis.

\section{Materials and Methods}

\section{Study Patients}

This study was approved by the institutional review board of Ege University Children's Hospital and was conducted during a 2-year period. The study of children younger than 24 months (excluding newborns) who were hospitalized with acute bronchiolitis was conducted between August 2015 and August 2017 at the General Pediatrics ward of the Children's Hospital. According to the recommendations of American Academy of Pediatrics guidelines, the diagnosis of acute bronchiolitis was based on at least 2 of the following signs: chest retractions, tachypnea, and wheezing or rales on auscultation following viral upper respiratory tract infection in children less than 24 months of age for the first time (13). Excluded were infants hospitalized within 2 weeks before the current admission, those who developed nosocomial acute bronchiolitis, or those who had a known history of bronchopulmonary dysplasia or chronic lung disease or congenital heart disease or any immunodeficiency. Infants who were born prematurely and with a known history of any chronic disease and hematologic diseases were also excluded from the study. Information regarding demographics, medical history, clinical characteristics and laboratory results were obtained from computerized hospital medical records. The ethics committee of Ege University approved the study (Number: E.155324). Infants who were hospitalized with acute bronchiolitis were recruited with informed, written, parental consent.

Data Acquisition, Blood Samples, and Detection of Respiratory Viruses

The age, gender, clinical findings, values of mean platelet, white blood cell count (WBC), C-reactive protein $(C R P)$, and lymphocyte percentage were recorded from each patients'chart at admission. Complete blood counts were performed for all patients on presentation using a commercially available analyzer (CELL-DYN Ruby, Abbott Park, Illinois, U.S.A). The reference range for MPV was between 4.3 and $13.2 \mathrm{fL}$. For accurate MPV measurement, the time between the blood sampling and MPV analysis was 1 hour, not more.

nasal smear was obtained from each infant and tested for the presence of RSV, influenza virus types $A$ and $B$, adenovirus, parainfluenza viruses, human rhinovirus, human coronavirus, human metapneumovirus, and human bocavirus with multiplex reverse-transcription PCR methods (RealAccurate, Respiratory RT PCR, PathoFinder, Netherlands, and Seeplex RV15 ACE Detection, Seegene, South Korea). Nasal samples were obtained more commonly by a nurse or sometimes a research assistant on all subjects within 48 hours of admission using a standardized protocol (14). Samples were frozen at $-20^{\circ} \mathrm{C}$ and transported in ice to the Department of Clinical Microbiology and Virology Laboratory of our university for viral nucleic acid amplification.

\section{Statistical Analysis}

Statistical analyses were performed using IBM SPSS version 21.0 for personal computers (Chicago, IL, USA). Group differences in categorical variables were analyzed using the Fisher Exact test. Shapiro-Wilk test was used to check the normality assumption of the continuous variables. In cases of non-normally distrubuted data, the Mann-Whitney $U$ test was used to determine whether the difference amongst the groups was statistically significant. The discriminative ability of MPV in respiratory viruses was evaluated by drawing receiver operating characteristic (ROC) curves for the biomarkers, and calculating their sensitivity, specificity for acute bronchiolitis. A value of $P<0.05$ was considered as statistically significant.

\section{Results}

In our study, the study group consisted of 184 patients with acute bronchiolitis, 114 (62\%) of them were male, 
and $70(38 \%)$ were female. The median age was $12 \pm 14.5$ months. One hundred twenty-six $(68.5 \%)$ children had at least one viral respiratory agent and the most common two viruses were RSV $(18 \% ; n=51)$ and rhinovirus $(16.2 \%$; $\mathrm{n}=46$ ). The distribution of respiratory viruses is shown in Figure 1 (Figure 1). RSV was the most common agent. The MPVs were similar in patients with positive and negative RSV bronchiolitis $(6.8 \pm 1.5$ vs. $7 \pm 1.3, P>0.05)$. The comparison of the lymphocyte percentage showed a significantly higher lymphocyte percentage in infants with RSV bronchiolitis $(P<0.05)$. There was no difference between the groups in terms of CRP $(P>0.05)$.

The differences that were found regarding the laboratory findings between patient groups with respiratory viruses are shown in Table 1. The MPV was significantly lower in patients with single RSV bronchiolitis versus their negative counterparts $(6.6 \pm 1.1$ vs. $7 \pm 1.2, P=0.04)$. The groups who had non-single RSV bronchiolitis tended to possess a statistically significant high WBC $(P=0.02)$. Being positive or negative in terms of respiratory viruses did not make any statistical difference in MPV and other laboratory findings.

ROC curve analysis suggested that cut-off of MPV level point for making the diagnosis of single RSV bronchiolitis was $6.63 \mathrm{fL}$ with a sensitivity, specificity of $55 \%, 63 \%$ respectively. The median AUC was 0.384 for the MPV (95 $\% \mathrm{Cl} 0.270-0.499, P=0.04)$. The AUC value for CRP (0.620) was higher than those of others such as MPV, WBC and lymphocyte percentage. The AUC values for MPV were the lowest (Table 2 and Figure 2).

\section{Discussion}

We have analyzed whether there is a clinical significance of MPV on the diagnosis and prediction of the RSV bronchiolitis and other respiratory pathogens. As we know, some cytokines and mediators are activated for reactionary responses in our body as a result of inflammatory conditions such as infections. It is also known that cytokines mediated by T-helper cells and other $\mathrm{T}$ cells provide exaggerated inflammation in the pathogenesis of acute bronchiolitis depending on the infective virus. The studies that investigated the differences of the laboratory markers of acute bronchiolitis have demonstrated a negative correlation between MPV and acute bronchiolitis $(11,12)$. A study performed in Turkey with the purpose to detect whether MPV could be used as a diagnostic tool for severe bronchiolitis has reported that the MPV was significantly lower statistically in patients with acute bronchiolitis compared to the healthy children; the

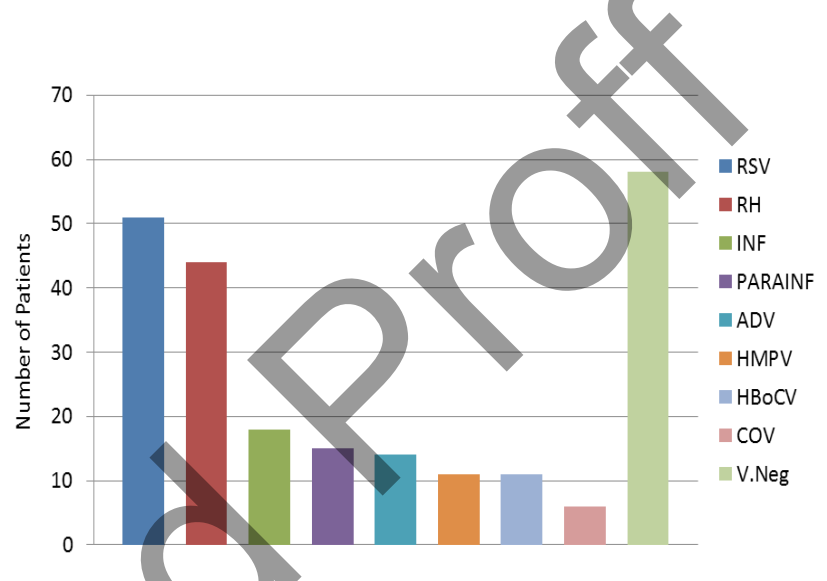

Figure 1. Dibtribution of respiratory viruses

RSV: Respiratory Syneytial Virus, RH: Rihinovirus, INF: Influenza virus, PARAINF: Parainfluenza virus, ADV: Adenovirus, HMPV: Human metapneumonia virus, HBoV: Human bocavirus, COV: Coronovirus. V.Neg: Virus Negative

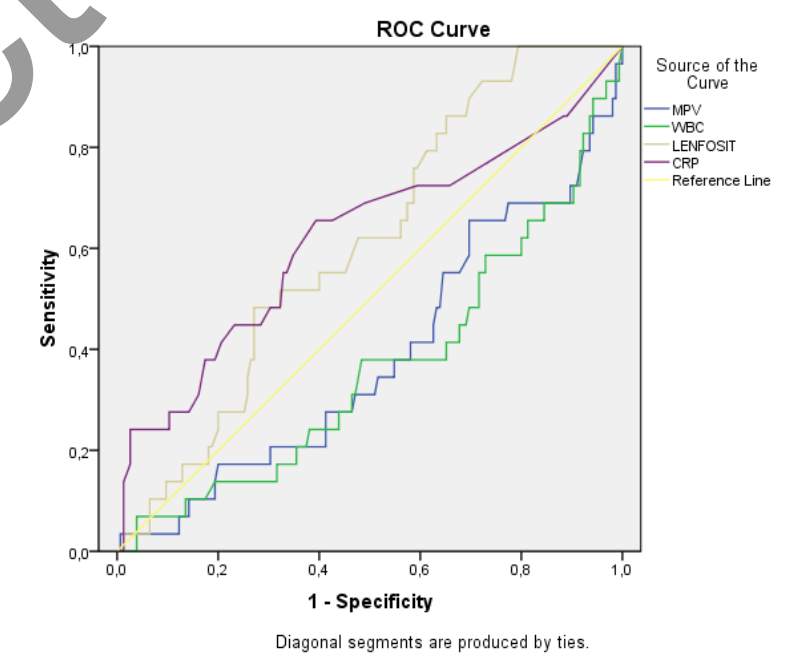

Figure 2. ROC curve of MPV, WBC and CRP count for single RSV bronchiolitis

Table 1. Comparison of laboratory data of the study groups diagnosed with Respiratory syncytial virus positive and negative bronchiolitis

\begin{tabular}{|l|l|l|l|}
\hline Parameters & RSV- positive cases $(\mathbf{n}=\mathbf{5 1})$ & RSV- negative cases $(\mathbf{n}=\mathbf{8 1})$ & $\mathbf{p}$ value \\
\hline MPV (med \pm IR) (f/lt) & $6.8 \pm 1.5$ & $7 \pm 1.3$ & NS \\
\hline WBC/mm (med \pm IR) & $8960 \pm 6630$ & $11800 \pm 5850$ & 0.00 \\
\hline Lymphocyte (med \pm IR) (\%) & $51 \pm 28$ & $34 \pm 31.5$ & 0.00 \\
\hline C-reactive protein (med \pm IR) (mg/dL) & $0.6 \pm 1.9$ & $0.4 \pm 1.6$ & NS \\
\hline
\end{tabular}

RSV: Respiratory syncytial virus, MPV: Mean platelet volume, WBC: White blood cell, CRP: C-reactive protein, NS: Not significant 
Table 2. Comparison of laboratory data of patients in terms of respiratory viruses groups

\begin{tabular}{|c|c|c|c|c|c|}
\hline Viruses & $\begin{array}{l}\text { MPV } \\
(\operatorname{med} \pm \mathrm{IR})(\mathrm{f} / \mathrm{It})\end{array}$ & $\begin{array}{l}\text { WBC } \\
(\operatorname{med} \pm \mathrm{IR}) / \mathrm{mm}^{3}\end{array}$ & $\begin{array}{l}\text { Lymphocyte } \\
\text { (med } \pm \text { IR) (\%) }\end{array}$ & $\begin{array}{l}\text { CRP } \\
(\operatorname{med} \pm \mathrm{IR})(\mathrm{mg} / \mathrm{dL})\end{array}$ & $p$ value \\
\hline \multicolumn{6}{|l|}{ RSV } \\
\hline $\begin{array}{l}\text { Single, }(n=29) \\
\text { Non single, }(n=97)\end{array}$ & $\begin{array}{l}6.6 \pm 1.1 \\
7 \pm 1.2\end{array}$ & $\begin{array}{l}8960 \pm 6420 \\
11760 \pm 6540\end{array}$ & $\begin{array}{l}51 \pm 30 \\
37.9 \pm 37\end{array}$ & $\begin{array}{l}0.9 \pm 4.3 \\
0.3 \pm 1.2\end{array}$ & $\begin{array}{l}0.04,{ }^{\circ} 0.02, \\
\text { cNS, d } 0.04\end{array}$ \\
\hline \multicolumn{6}{|l|}{ Rhinovirus } \\
\hline $\begin{array}{l}\text { Positive, }(n=46) \\
\text { Negative, }(n=28)\end{array}$ & $\begin{array}{l}7 \pm 1.2 \\
6.8 \pm 1.3\end{array}$ & $\begin{array}{l}12690 \pm 6602 \\
1100 \pm 5110\end{array}$ & $\begin{array}{l}29 \pm 31.2 \\
44 \pm 30\end{array}$ & $\begin{array}{l}0.3 \pm 1.6 \\
0.6 \pm 1.9\end{array}$ & $\begin{array}{l}\text { aNS, bNS } \\
\text { o.01, dNS }\end{array}$ \\
\hline \multicolumn{6}{|l|}{ Adenovirus } \\
\hline $\begin{array}{l}\text { Positive, }(n=18) \\
\text { Negative, }(n=60)\end{array}$ & $\begin{array}{l}7 \pm 0.9 \\
6.9 \pm 1.4\end{array}$ & $\begin{array}{l}11900 \pm 6375 \\
11220 \pm 6092\end{array}$ & $\begin{array}{l}41 \pm 50.5 \\
40.4 \pm 34.2\end{array}$ & & $\begin{array}{l}\text { aNS, }{ }^{b} 0.03 \\
\text { cNS, d NS }\end{array}$ \\
\hline \multicolumn{6}{|l|}{ Virus } \\
\hline $\begin{array}{l}\text { Positive, }(n=136) \\
\text { Negative, }(n=58)\end{array}$ & $\begin{array}{l}6.9 \pm 1.3 \\
6.9 \pm 1\end{array}$ & $\begin{array}{l}11300 \pm 5300 \\
12300 \pm 7500\end{array}$ & $\begin{array}{l}40 \pm 37 \\
35 \pm 34\end{array}$ & $\begin{array}{l}0.4 \pm 1.7 \\
0.3 \pm 1\end{array}$ & $\begin{array}{l}\text { aNS, bNS } \\
\text { cNS, dNS }\end{array}$ \\
\hline
\end{tabular}

MPV: Mean platelet volume, WBC: White blood cell, CRP: C-reactive protein, RSV: Respiratory syncytial virus, NS: Not significant, a: Shows the statistical difference of MPV in virus groups, b: Shows the statistical difference of WBC in virus groups, $c$ : Shows the statistical difference of lymphocyte percentage in virus groups, d: Shows the statistical difference of CRP in virus groups.

Table 3. Results of the receiver operating characteristic curve of MPV, CRP, WBC and Lymphocyte for Single RSV bronchiolitis

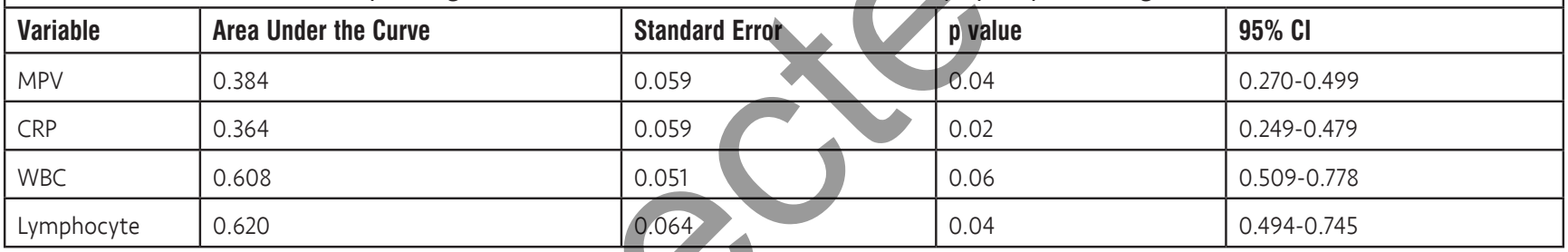

WBC: White blood cell, CRP: C-reactive protein, MPV: Mean plateletvolüme, RSV: Respiratory syncytial virüs, Cl: Confidence interval

A p value of $<0.05$ was considered statistically significant

authors of this study explain the pathophysiology of the low mean platelet value as associated with the migration of large young platelets to the site of inflammation and hence a relative decrease in the circulation (11). Our current study of the infants hospitalized with bronchiolitis has shown that the respiratory pathogens which cause acute bronchiolitis cannot be diagnosed or predicted by mean platelet volume. Our results indicated that bronchiolitis with RSV was associated with a reduced MPV which was not statistically significant. However, we demonstrated that the single RSV had lower MPV compared to non-single RSV bronchiolitis with an MPV under $6.63 \mathrm{fl}$ that was relatively sensitive and specific for the single RSV infection. Similar to our study, Renshow AA et al. reported that MPV was lower in patients hospitalized with RSV compared to the control group by rapid RSV assays and viral cultures in 158 patients, 112 of which were aged $<18$ years (12). In this study, it is also reported that the MPV under 8.9 $\mathrm{fl}$ with a sensitivity of $71 \%$ and specificity of $49 \%$ was to be a useful marker in children undergoing bronchoscopy in RSV bronchiolitis.

Regarding the systematic review of current literature for mean platelet volume and pediatrics, we searched for articles published up to 2017 in the following databases: Pubmed, Medline, Embase, Cochrane libraries, and CINAHL. We used search terms including "mean platelet volume," "pediatrics," to identify reports that presented data on these topics. In Pubmed, when we researched the topics, more than 100 articles appeared. However, topics of mean platelet volume, pediatrics and acute bronchiolitis together were found in only one article conducted by Ergül et al (11). Although the MPV measurements in autoimmune, cardiac conditions and most infections were reported in adult studies, there is a lack of information on MPV for pediatric patients with acute bronchiolitis diagnosed with RSV or other respiratory viruses.

Actually, the current study of the changes of MPV has proved that there is no relevance between respiratory viruses and MPV. However, the results obtained from our study have shown that infants with single RSV bronchiolitis have significantly decreased MPVs compared to those with RSV accompanied by other respiratory viruses. Thus we could recommend that the effect of single RSV on MPV might be specific to this virus condition and MPV may be a useful predictor for diagnosing single RSV bronchiolitis. 
Some factors should be taken into account for accurate MPV assessment like anticoagulant type and time interval between blood sampling and MPV analysis. MPV increases over time in EDTA (Ethylenediaminetetraacetic acid) tube and shows an increase up to $7.9 \%$, and over $24 \mathrm{~h}$ MPV increases overall up to $13.4 \%$ (15). Due to the fact that the standardized methods should be used in MPV measurement, all measurements were made at the same hospital on the same analyzer, and specimens were received and processed in our study.

There are several limitations to this study. We only investigated the hospitalized patients with acute bronchiolitis, and not the infants who applied to the emergency services or out-patients policlinics, so our study group was small in number. Prospective studies with larger number of patients are needed to assess the role of MPV values in acute bronchiolitis. Although the assessment of MPV was not associated with extra cost or effort, we ignored the conditions that could affect the measurement, such as room temperature. We were not able to document the association between patient admission and the time of onset of symptoms. We thought that MPV could have changed over the course of the illness. Finally, it is possible that some subjects may have had infection with other possible agents such as atypical or bacterial pathogens. We have also ignored this situation, but just focused on the respiratory viruses.

\section{Conclusions}

The mean platelet volume may be utilized for the diagnosis of single RSV bronchiolitis. However, we think that MPV is not a reliable marker in specifying the cause of acute bronchiolitis; that its diagnosis could simply be made by conventional microbiological methods. The supportive testing of bronchiolitis, for example complete blood cell (CBC) counts, should be utilized for ruling out other diagnoses but, $C B C$ counts are poor test characteristics for determining the cause of the disease. The studies report that $C B C$ data can not demonstrate the diagnosing of bronchiolitis nor used as guiding therapy. In any case, the diagnosis of bronchiolitis is based primarily on typical history and results of a physical examination without blood analysis. Rather than guessing the virus type according to laboratory findings, we should use methods that will minimally hurt the patient.

\section{Declaration of Conflicting Interests}

The author(s) declared no potential conflicts of interest with respect to the research, authorship, and/or publication of this article.

\section{Funding}

The author(s) received no financial support for the research, authorship, and/or publication of this article.

\section{Author Contributions}

ŞG: Contributed to conception and design; contributed to analysis; drafted the manuscript; gave final approval; agrees to be accountable for all aspects of work ensuring integrity and accuracy. ŞG, ZK, GK also researched literature and conceived the study. ZK, CK, SB, CC was involved in protocol development, gaining ethical approval, patient recruitment and data analysis: Drafted the manuscript. ŞG, SP: Contributed to conception and design, and data collecting, CÇ: Contributed to acquisition, analysis, or interpretation. All authors reviewed and edited the manuscript and approved the final version of the manuscript

\section{Acknowledgments}

The authors would like to thank the study staff at the Microbiology Laboratory for conducting the study and the infants and their families for participating in the study. In addition, we wish to formally acknowledge Dr Aslı Süner who helped the statistical analyses.

List of abbreviations: AUC, area under the curve; $\mathrm{CBC}$, complete blood cell; CRP, C-reactive protein; EDTA, Ethylenediaminetetraacetic acid; MPV, mean platelet volume, RSV, Respiratory syncytial virus; ROC, receiver operating characteristic; WBC, white blood cell count.

\section{References}

World Health Organization. WHO Consultation on respiratory syncytial virus (RSV) vaccine development. http://www.who. int/immunization/research/meetings_workshops/rsv_vaccine development/en/(Accessed 5 June, 2017).

2. World Health Organization. Research and Development. http:// www.who.int/immunization/research/meetings workshops/ rsv_vaccine_development/en/(Accessed 5 June 5, 2017).

3. Black-Payne C. Bronchiolitis, in: Hilman BC. (Eds.), Pediatric Respiratory Disease: Diagnosis and Treatment, Philadelphia, PA: WB Saunders,1993, pp. 205-218.

4. Berry M, Gamieldien J, Fielding BC. Identification of new respiratory viruses in the millennium. Viruses. 2015; 7:996-919.

5. Morrell CN, Aggrey AA, Chapman LM, Modjeski KL. Emerging roles for platelets as immune and inflammatory cells. Blood. 2014;123: 2759-2767.

6. Bilici S, Sekmenli T, Goksu M, Melek M, Avci V. Mean platelet volume in diagnosis of acute appendicitis in children. Afr Health Sci. 2011; 11: 427-432.

7. Beyazit $Y$, Sayilir A, Torun S, et al. Mean platelet volume as an indicator of disease severity in patients with acute pancreatitis. Clin Res Hepatol Gastroenterol. 2012;36:162-168.

8. Karadag-Oncel E, Ozsurekci Y, Kara A, Karahan S, Cengiz $A B$, Ceyhan $M$. The value of mean platelet volume in the determination of community acquired pneumonia in children. Italian Journal of Pediatric. 2013;39:16.

9. Sahin F1, Yazar E, Yıldız P. Prominent features of platelet count, plateletcrit, mean platelet volume and platelet distribution width in pulmonary tuberculosis. Multidisciplinary Respiratory Medicine. 2012;7:38. 
10. Tozkoparan E, Deniz O, Ucar E, Bilgic H, Ekiz K. Changes in platelet count and indices in pulmonary tuberculosis. Clin Chem Lab Med. 2007;45:1009-1013.

11. Ergül AB, Torun YA, Uytun S, Aslaner H, Kısaaslan AP, Şerbetçi $M C$. Reduction in mean platelet volume in children with acute bronchiolitis. Turk Pediatri Ars. 2016;51: 40-45.

12. Renshaw AA, Drago B, Toraya N, Gould EW. Respiratory syncytial virus infection is strongly correlated with decreased mean platelet volume. International Journal of Infectious Diseases. 2013;17:678-680.
13. American Academy of Pediatrics Subcommittee on Diagnosis and Management of Bronchiolitis, Diagnosis and management of bronchiolitis. Pediatrics. 2006;118:1774-1793.

14. Bezerra PG, Britto MC, Correia JB, et al. Viral and atypical bacterial detection in acute respiratory infection in children under five years. PLoS One. 2011;6:18928.

15. Lance' MD, Sloep $M$, Henskens YM, Marcus MA. Mean platelet volume as a diagnostic marker for cardiovascular disease: drawbacks of preanalytical conditions and measuring techniques. Clin Appl Thromb Hemost. 2012;18:561-568.

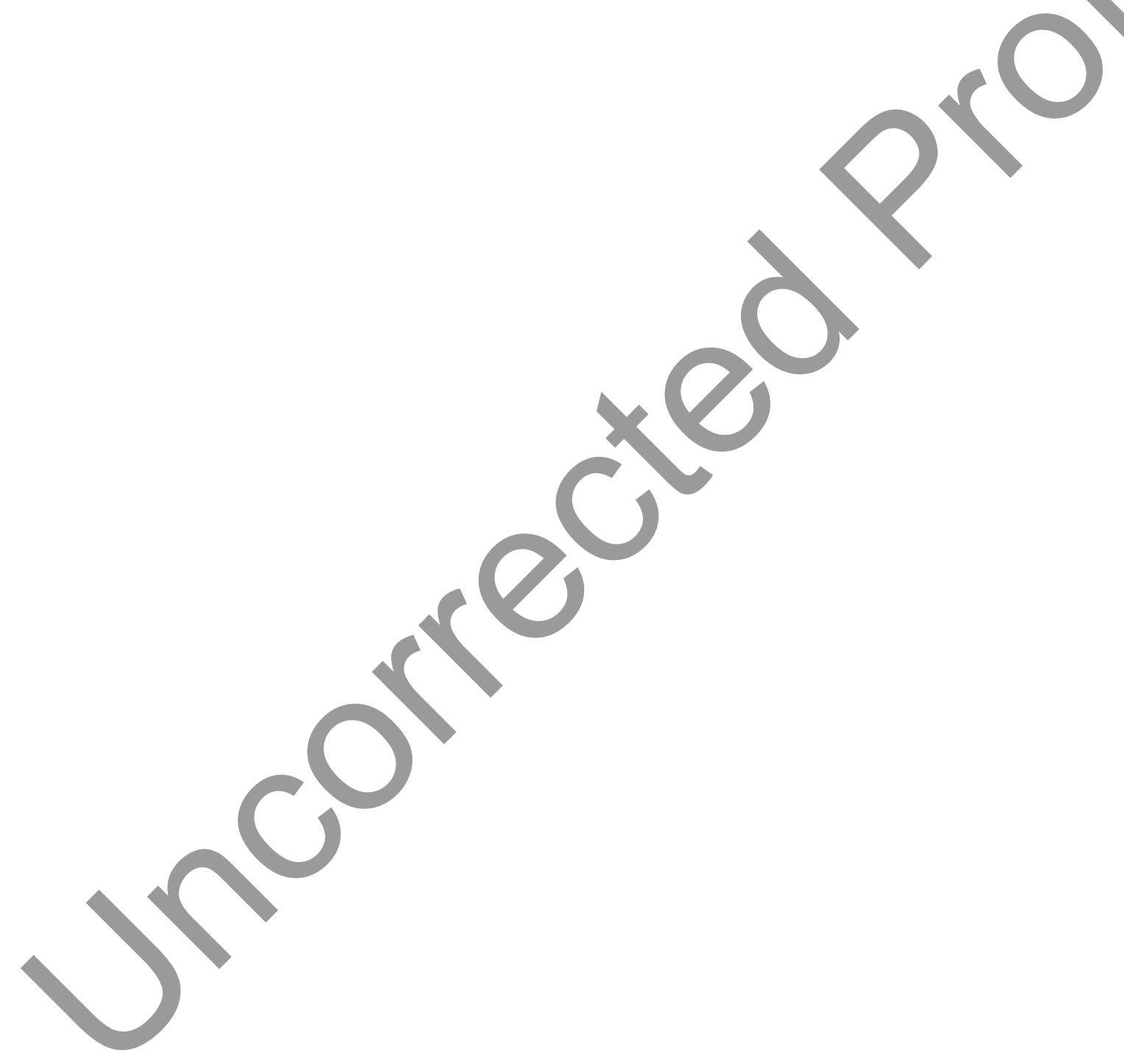

